\section{The current status of and measures against maternal suicide in Japan}

\author{
Satoru Takeda ${ }^{1,2}$ \\ ${ }^{1}$ Department of Obstetrics and Gynecology, Faculty of Medicine, Juntendo University, \\ Tokyo, Japan, ${ }^{2}$ Aiiku Research Institute, Tokyo, Japan
}

\section{Reprint request to:}

Satoru Takeda, M.D., Ph.D., Department of Obstetrics and Gynecology, Juntendo University, 2-1-1 Hongo, Bunkyoku, Tokyo 113-8421, Japan.

E-mail: stakeda@juntendo.ac.jp

\section{Key words:}

maternal mortality, maternal suicide, perinatal mental healthcare

Received: April 27, 2020

Revised: July 10, 2020

Accepted: July 14, 2020

J-STAGE Advance published date: August 22, 2020

DOI:10.14390/jsshp.HRP2020-005

Rapid progress in perinatal care in recent decades has led to a dramatic decline in perinatal, neonatal, and maternal mortality (excluding suicides), and achieved remarkable improvements in obstetrical outcomes in Japan. However, while maternal mortality had been on a continuous and steady decline up until 2007 (3.1/100,000 total births), the rate has been fluctuating since then (e.g., 2.7/100,000 in 2014, 3.4/100,000 in 2016). This is likely attributed to a variety of factors that have emerged in the past 20 years due to changes in the environment and social situation surrounding women, such as later marriage and rise in maternal age.

In Western countries, "late maternal deaths" occurring between 42 days and one year after delivery are considered to be just as important as "maternal deaths," i.e., deaths during pregnancy or within 42 days of termination of pregnancy. In particular, suicides attributable to psychiatric disorders have become a serious issue among women less than one year postpartum. However, in Japan, the actual number of deaths by suicide is unknown, since neither death certificates nor postmortem certificates include information on pregnancy and delivery. Despite the fact that the total number of suicide deaths in Japan is known, whether such deaths are associated with perinatal mental issues or not is unclear, and thus, no measures have been taken. Untreated perinatal depression and psychiatric disorders not only cause issues such as suicide, but are also related to pediatric developmental and mental disorders, neglect, and/or child abuse due to impaired nurturing ability. Suicide rates among pregnant and parturient women in Osaka, Tokyo, and Mie are much higher than those of the UK, the US, and Sweden. There is an urgent need to establish a regional support system that facilitates interactions among the obstetrical, pediatric, psychiatric field, and local administrations for monitoring and supporting mothers and infants, as well as a system that allows families, schools, and society to support young people, in order to realize improved preconception health care.

\section{Introduction}

Maternal and child health statistical indicators, such as perinatal, neonatal, and maternal mortality rates, are low in Japan, and reportedly rank in the top group in the world. However, until recently, statistics of maternal deaths did not include cases of suicide due to postpartum depression or other mental disorders in Japan. Thus, a simple comparison with other countries is not feasible. In 2017, suicide cases due to mental disorders during pregnancy and within one year after delivery began to be included in the statistics of maternal deaths in Japan. Death certificates and postmortem certificates often lack information pertaining to events in pregnancy or puerperium. For this reason, it is difficult to determine whether the cause of death (i.e., disease) was influenced by pregnancy or delivery. This renders the accuracy of maternal death statistics questionable, and the actual status of suicides among pregnant, parturient, and puerperal women, and the reality of late maternal deaths, remain unclear. In recent years, results of regional and national surveys regarding suicide among pregnant, parturient, and puerperal women have shed light on the status of underlying postpartum depression. Since the frequency of suicide among pregnant, parturient, and puerperal women in Japan is markedly higher compared 
to other countries, urgent measures are necessary. ${ }^{1)}$

In 1991, a prospective long-term large-scale cohort study in the UK (Avon Longitudinal Study of Parents and Children: ALSPAC) was initiated, and followed approximately 14,000 pregnant women from the prenatal period to roughly 18 years postpartum. ${ }^{2)}$ Findings from that study, which have been published sequentially, attracted much attention. With respect to parent-child relationships, prenatal anxiety and depression and postpartum depression in parents were associated with developmental disorders and attention/ behavior problems in children. Moreover, prenatal and postpartum depression was associated with decreased IQ and depression in children. These results suggest that the antepartum and postpartum mental status of mothers greatly affects the mental development and growth of children, highlighting the importance of perinatal mental health care in mothers.

In these social situations, perinatal mental health measures are urgently needed to improve the child-rearing environment and promote the growth and development of mentally and physically healthy children who form the next generation. To address this important issue, not only healthcare professionals, but also administrative staff, welfare service providers, and citizens will need to work together to understand the actual condition, share problems, and devise solutions.

\section{Importance of statistics of late maternal deaths}

Maternal mortality (i.e., the rate of maternal deaths during pregnancy and within 42 days after delivery) in Japan has plateaued since 2007, fluctuating in the range of 2.7-4.8 deaths per 100,000 deliveries ${ }^{2,3)}$ in recent years. On the other hand, late maternal mortality (i.e., the rate of maternal deaths between 42 days and one year after delivery), which covers the period during which suicide rates due to postpartum depression are high, has rarely been reported until 2016. This is because suicides due to mental disorders, such as depression, had not been included in maternal mortality statistics prior to 2016. However, maternal and child health statistics overseas suggest that the number of late maternal deaths is higher than that of early maternal deaths, with the predominant cause of death being suicide. Since maternal and child health statistics from 2017 on include information from death certificates and postmortem certificates, suicide may be recognized in the future as the predominant cause of maternal and late maternal mortality.

\section{Comparison of perinatal suicide rates between Japan and overseas}

Direct obstetric deaths are more common than indirect deaths in Japan, with the ratio being 3:1. Indirect deaths are more common in Europe and North America than in Japan. In the UK, the maternal mortality rate in 20132015 was $8.8 / 100,000$ deliveries, ${ }^{4)}$ which was higher than that in Japan, and there was a decrease in direct obstetric mortality with no change in indirect obstetric mortality, resulting in a higher proportion of indirect obstetric deaths. The late maternal mortality rate was higher at 14.1/100,000 deliveries (data not available in Japan). The predominant cause of late maternal deaths was malignant tumor $(24 \%)$, followed by suicide related to mental health (14\%). During the period of 2003-2015, suicide continued to be the leading cause of direct deaths in pregnant, parturient, and puerperal women, for both maternal and late maternal mortality. The suicide rate was $2.5 / 100,000$ deliveries, which can be broken down to $0.52 / 100,000$ deliveries among pregnant women and those within six weeks after delivery, and 1.98/100,000 deliveries among puerperal women (between six weeks and one year after delivery). ${ }^{4}$ As is evident from these figures, suicide has been the most pressing issue in the UK, requiring countermeasures among the various causes of death in pregnant, parturient, and puerperal women. ${ }^{4)}$ In the UK, suicide due to mental disorders is classified as a direct obstetric death, while deaths due to drugs or alcohol are included among indirect obstetric deaths. In Japan, however, both types of death are classified as indirect obstetric deaths. The World Health Organization (WHO) recommends that suicide due to mental disorders be classified as a direct obstetric death, but this classification has not yet been generally adopted. Indeed, suicide due to mental disorders is classified as an indirect obstetric death in Australia, or as an incidental death in some countries. Efforts should be made to unify these classifications in the future.

In Japan, national statistics have yet to clarify the current status of suicide. However, annual mortality rates from suicide during pregnancy or within one year after delivery (sum of deaths during pregnancy and late maternal deaths) were $8.7 / 100,000$ deliveries in the 23 wards of Tokyo (2005-2014), ${ }^{1)} 13.0 / 100,000$ deliveries in Osaka City (2012-2014), and 14.1/100,000 deliveries in Mie Prefecture (2013-2014). Since the number of deliveries in Tokyo accounts for roughly $10 \%$ of all deliveries in Japan, the number of suicides among pregnant, parturient, and puerperal women in Japan is estimated to be roughly 80-90 annually. According to data in Osaka City as well, the annual number of suicides in Japan is estimated to be $60-80$. These regional surveys on suicide among pregnant, parturient, and puerperal 
women in Japan suggest an extremely high frequency of suicide in comparison with other countries, including the UK (2.3/100,000 deliveries) and Sweden (3.7/100,000 deliveries) ${ }^{5,6)}$ A two-year national survey conducted by Mori et al. at the Ministry of Health, Labour and Welfare revealed that the most common cause of maternal deaths within one year after delivery was suicide in 2015-2016, followed by cancer. ${ }^{7)}$ Similar to the UK, the number of late maternal deaths in Japan was higher compared to maternal deaths, with suicide being the most frequent cause. Thus, there is an urgent need for measures in Japan as well.

According to statistics of pregnant, parturient, and puerperal women in 7-17 states in the US (2003-2007), ${ }^{8)}$ homicides were the most frequent cause of death, at a rate of 2.9/100,000 deliveries, and suicide was the second most frequent cause (2.0/100,000 deliveries), followed by bleeding, pregnancy induced hypertension, and amniotic fluid embolism. According to state-level statistics in the US, the mortality rate among pregnant, parturient, and puerperal women in North Carolina $(2004-2006)^{9)}$ was 2.9/100,000 pregnancies for homicide and 2.7/100,000 pregnancies for suicide, and 4.6/100,000 deliveries for suicide in Colorado (2004-2012). ${ }^{10)}$ In Canada (2002), ${ }^{11)}$ the rate of suicide in puerperal women within one year after delivery was $0.7 / 100,000$ deliveries. Specifically, in Ontario, Canada (1994-2008), ${ }^{12)}$ suicide occurred at a rate of 2.6/100,000 deliveries. In Italy (2006-2012), ${ }^{13)}$ the corresponding rate was $2.30 / 100,000$ deliveries $(1.18$ after delivery, 2.77 after induced abortion, and 2.90 after spontaneous abortion) (Figure 1).

In Finland (1987-1994), ${ }^{14)}$ the rate of suicide among puerperal women after delivery was $11.3 / 100,000$ deliveries. Specifically, the rate per 100,000 deliveries was 18.1 after spontaneous abortion and 34.7 after induced abortion, revealing a higher rate of suicide after induced abortion. According to newer statistics on suicide in Finland (2001-2012), ${ }^{15)}$ the rate of suicide in pregnant, parturient, and puerperal women decreased to $3.3 / 100,000$ pregnancies. Notably, the rate of suicide was 10.2/100,000 non-pregnant women, and the rate of suicide after induced abortion was higher, with a rate of 21.8/100,000 deliveries. According to an Australian report $(2006-2010),{ }^{16)}$ the rate of suicide during pregnancy was $0.9 / 100,000$ pregnancies, whereas the rate of suicide among age-matched non-pregnant women was 6.0/100,000 non-pregnant women. Thus, there is a consistent trend in Japan and other countries of suicide being less frequent during pregnancy and puerperium. In Taiwan $(2002-2012),{ }^{17)}$ the rate of suicide among puerperal women within one year after delivery was $6.86 / 100,000$ deliveries on average, showing a decrease after 2010 (Figure 1). Since suicide occurs more frequently in the early pregnancy period, as evidenced by data from Tokyo, unwanted pregnancy and induced abortion are considered major risk factors for suicide.

\begin{tabular}{|c|c|c|c|}
\hline $\begin{array}{l}\text { City/ } \\
\text { Country }\end{array}$ & $\begin{array}{l}\text { Maternal } \\
\text { mortality rate } \\
\text { (per 100,000 live birth) }\end{array}$ & $\begin{array}{l}\text { Maternal } \\
\text { suicide rate } \\
\text { (per 100,000 live birth) }\end{array}$ & Survey period \\
\hline Tokyo & & 8.7 & $2005-2014$ \\
\hline Osaka & & 13.0 & $2012-2014$ \\
\hline Mie & & 14.1 & $2013-2014$ \\
\hline Japan & $\begin{array}{l}3.8 \\
\text { (2015; excluded suicide) }\end{array}$ & & \\
\hline UK \& NI & $9.0(2011-13)$ & $\begin{array}{l}2.3 \\
\text { (per } 100,000 \text { matenities) }\end{array}$ & $2009-2013$ \\
\hline Sweden & $6(1988-2007)$ & 3.7 & $1980-2007$ \\
\hline USA ( $7-17$ states) & & 2.0 & $2003-2007$ \\
\hline North Carolina & & 2.7 & $2004-2006$ \\
\hline Colorado & & 4.6 & $2004-2012$ \\
\hline Ontario, Canada & & 2.6 & $1994-2008$ \\
\hline Italy & & 2.30 & $2006-2012$ \\
\hline Finland & & 3.3 & $2001-2012$ \\
\hline Australian & $7.1(2008-2012)$ & 0.9 & $2006-2010$ \\
\hline Taiwan & & 6.86 & $2002-2012$ \\
\hline
\end{tabular}

Figure 1. Comparison of maternal suicide rate (during pregnancy and one year postpartum). 


\section{Suicide in pregnant, parturient, and puerperal women with mental disorders}

The prevalence of mental disorders in pregnant and parturient women in community maternity hospitals in Japan was reported to be $6.2 \%{ }^{18)}$ Among 355 institutions registered in the Japan Society of Obstetrics and Gynecology registry project (2014), the rate of pregnancy accompanied by mental disorders was $2.5 \%$ in 2014 ; this rate has been fluctuating in the range of $2.5 \%-2.8 \%$ since then. Mental disorders related to suicide in pregnant, parturient, and puerperal women include depression, puerperal psychosis, bipolar disorder, schizophrenia, and anxiety disorder. In the UK, $46 \%$ of people who committed suicide had a history of psychiatric treatment. According to a survey in Tokyo, 39\% of people who committed suicide during pregnancy had a history of psychiatric disorders; the frequency of depression was $35 \%$, while the frequency of depression plus schizophrenia was $4 \%$. Of the women who committed suicide after delivery, $17 \%$ had a history of psychiatric disorders, including depression, schizophrenia, and depression combined with schizophrenia, in descending order. Postpartum depression was noted in 33\% of women who had no history of psychiatric disorders. Suicide victims with no record of psychiatric disorders might have included a substantial number of women with depression, as it is possible they did not see psychiatrists and thus were not diagnosed.

\section{Strategies to prevent suicide among pregnant, parturient, and puerperal women}

\section{1) Cooperation among obstetricians, psychiatrists, pediatricians, and local community}

Untreated perinatal depression and mental disorders not only affect the patients themselves, but can also lead to developmental disorders and mental retardation in children due to insufficient nurturing ability, neglect, and child abuse. To address this situation, a guideline was developed under the leadership of the Japanese Society of Perinatal Mental Health to raise awareness. ${ }^{3)}$ The legislative bill of postpartum care law was passed last year to secure support for establishing "comprehensive support centers" by 2020, with the aim of providing mental and physical care and child-rearing support for women and infants within one year after delivery, and to help mothers engage in child-rearing activities. With this bill, municipal governments are now required to implement postpartum care programs.

Mothers with high-risk factors for perinatal mental health should be followed during pregnancy and in the postpartum period by obstetricians, psychiatrists, nurses, midwives, and clinical psychologists. High-risk factors include psychosocial factors (e.g., unwanted pregnancies, unmarried mothers, post-abortion psychosocial risks, history of domestic violence, family rifts, history of self-injury, husband's incomprehension, economic problems) and anxiety and stress related to pregnancy and delivery (e.g., prolonged hospitalization, pregnancy with complications, anxiety concerning drug treatment, anxiety and fear over delivery and pain, posttraumatic stress disorder from past pregnancy and delivery, mother-infant separation). It is important for medical institutions, public health nurses, and the local administration to work in close cooperation to comprehensively support postpartum women in addressing conditions such as depression, and to provide support for child-rearing in general. ${ }^{19)}$ Follow-up for puerperal women by obstetricians usually ends one month after delivery. Accordingly, puerperal women who have a high Edinburgh Postnatal Depression Scale score, for example, or those with a history of mental disorder, should be followed in close cooperation with the local administration and public health nurses. ${ }^{19)}$

Mothers and children visit a local health center, public health center, or pediatric clinic for preventive vaccination. Such occasions can be used as opportunities to discuss with mothers not only the physical condition of their children, but also their own mental and physical conditions. This could lead to early intervention, although cooperation of pediatricians may be necessary. Cooperation with psychiatrists to learn more about the patient's mental disorder, and with doctors who treated the patient to implement careful observation of the therapeutic process from two perspectives, will also be important. If a postpartum woman has suicidal ideation/ suicidal thoughts, it is a matter of urgency. The patient should be brought to psychiatric emergency care in cooperation with obstetric care facilities, social workers, local public health nurses, and mental health welfare centers, while establishing a framework of support together with the patient's family. It is also important to cooperate with psychiatrists and clinical psychologists to formulate a system that protects and supports mothers and children in each area. Such a system should provide support to mothers and children not only for dealing with mental health-related issues, but also with their daily living (e.g., economic and manpower issues). Attention should also be paid to bonding between the mother and child, lactation, and affirmation of child-rearing.

Fathers, families, and the general public need to be informed of what postpartum depression is and how they can cope with it. To do so, a framework for society as a whole is necessary, which aims to maintain protective supervision over mothers and children. Given that one in 10 postpartum women develops postpartum depression, though of varying severity, it is important to have in place 
a system that facilitates counseling and consultation when the mother shows any sign of change in behavior and attitude. Giving a push to depressed postpartum women works negatively. The importance of educating people to listen and respond to patients in a sympathetic manner should be emphasized.

Cases of suicidal ideation or suicidal depression require an urgent response. A framework should be developed to support patients in cooperation with their family and to facilitate coalition building among obstetric institutions, social workers, community health nurses, and mental health welfare centers for directing patients to psychiatric emergency care. If patients have regular access to a psychiatric care facility, they are referred to that facility; if not, they should use psychiatric emergency services in the community.

\section{2) Human resource development and education}

Another matter of concern is the capability of personnel, assuming a child-rearing support system is in place. Thus, education for personnel represents an issue of importance. It is necessary to cultivate personnel who have sufficient knowledge, communication skills, and interview techniques, and who can work with problem awareness with great enthusiasm. It is common for depression due to adjustment disorder to improve or resolve when the child-rearing environment is modified. Maternal mental health issues are not only related to medical care but also involve various problems such as economic concerns and issues related to the childrearing environment and support. Patients should not simply be referred to psychiatrists; rather, various health professionals should work collaboratively and contribute their expertise in their own settings. Nurses, midwives, and public health nurses whom patients with maternal health issues first encounter should acquire skills, such as attentive listening and empathic responses, through various training courses and workshops. For example, there are workshops held by the Japanese Society of Perinatal Mental Health, the Japan Association of Obstetricians and Gynecologists, ${ }^{3,19)}$ as well as perinatal mental health professional training offered by the Kitamura Foundation for Studies and Skill Advancement in Mental Health and the Psychiatric Evaluation in Emergency Care (PEEC) educational course.

\section{3) Preconception health care}

The health and nutritional status of mothers before and during pregnancy influences the development and growth of fetuses and neonates, as well as the health status of children in adulthood (e.g., risk of metabolic syndrome) ${ }^{20-22)}$ The ALSPACA study, ${ }^{2)}$ a large-scale prospective birth cohort study in the UK, found that untreated perinatal depression and mental diseases not only affect the patients themselves, but also lead to development disorders and impaired mental development in children, neglect, and child abuse, due to the impaired ability of mothers to engage in child-rearing activities. It is a well-known fact that obesity and the lean before pregnancy, and maternal weight gain during pregnancy, affect the birth weight of neonates, and child birth weight influences the occurrence of metabolic syndrome in later stages of life. Smoking and excessive alcohol drinking affect pregnancy and delivery, as well as fetal weight. Low folate intake affects the development of neural tubes in the fetus and is associated with spina bifida and anencephaly of the fetus. ${ }^{23)}$ An association between low mineral and vitamin intake during pregnancy and impaired fetal development has also been reported. ${ }^{24-27)}$ Thus, it is apparent that pre-pregnancy health and nutritional status, exercise, and lifestyle of parents have a great impact on the course of pregnancy and delivery, the health status of the mother and child, mental and physical development of the child, and the health status of the child in adulthood. In this context, preconception health care has become recognized as an important topic.

Pre-pregnancy women should pay attention to their mental and physical health, nutrition, and exercise, and reconsider and improve their lifestyle habits, as these factors greatly affect the growth and health status of children during gestation and in later stages, and are also associated with the development of metabolic syndrome in adulthood. Education should be provided regarding this fact, be aware of it, and practice it, as this would help build a wholesome society through maintenance and enhancement of health of the next generation. Schools play an important role in the provision of sex education, including information on contraceptive methods and morning-after pills to avoid unwanted pregnancy, which is important in view of preventing suicide in early pregnancy. Young people of child-bearing/rearing age should lead an independent life, with the ability to plan an appropriate timing for pregnancy and delivery. Efforts to develop supportive family-, school-, and society-based systems for young people should be made in order to realize improved preconception health care. Key points of maternal and child health policies are to facilitate pregnancy, delivery, and child-rearing under mentally and physically healthy conditions, and to promote mental and physical health of children who will form the next generation.

\section{4) Future prospects}

As seen in countries such as Finland and Taiwan, where approaches to the issue of suicide in pregnant, parturient, and puerperal women have made considerable progress, establishment of a support system that provides maternal and child mental and physical care and child-rearing 
support is important for helping mothers engage in child rearing activities. Support for daily living, which encompasses economic and physical aspects, is also important in the management of maternal mental health issues. Attention should also be paid to mother-child bonding, breast-feeding behaviors, and development of child-rearing skills. It is only when the importance of perinatal mental health care is properly advocated and recognized that major progress is achieved. Steady efforts of all people involved are important to this end.

\section{Conflict of interest}

None.

\section{References}

1. Takeda S, Takeda J, Murakami K, et al. Annual Report of the Perinatology Committee, Japan Society of Obstetrics and Gynecology, 2015: Proposal of urgent measures to reduce maternal deaths. J Obstet Gynecol Res. 2017; 43: 5-7.

2. Avon Longitudinal Study of Parents and Children. http://www. bristol.ac.uk/alspac/ accessed date December 17, 2019.

3. Takeda S. Education and training approaches for reducing maternal deaths in Japan. Hypertension Research in Pregnancy. 2018; 6: 15-19.

4. Saving Lives. Improving Mother's Care-Surveilance of maternal deaths in the UK and lessons learned to inform maternity care from the UK and Ireland Confidential Enquiries into Maternal Deaths and Morbidity 20013-15; MBRRACE-UK, 2017.

5. Saving Lives. Improving Mothers' Care - Surveillance of maternal deaths in the UK 2011-13 and lessons learned to inform maternity care from the UK and Ireland Confidential Enquiries into Maternal Deaths and Morbidity 2009-13; MBRRACE-UK, Dec 2015.

6. Esscher A, Essén B, Innala E, et al. Suicides during pregnancy and 1 year postpartum in Sweden, 1980-2007. Br J Psychiatry. 2016; 208: 462-469.

7. Mori R. Survey finds suicide is leading cause of maternal death in Japan. https://www.japantimes.co.jp/news/2018/09/07/national/ science-health/survey-finds-suicide-leading-cause-maternal-deathjapan/\#.XqUkg25uJdg. accessed date April 26, 2020.

8. Palladino CL, Singh V, Campbell J, et al. Homicide and suicide during the perinatal period: Findings from the national violent death reporting system. Obstet Gynecol. 2011; 118: 1056-1063.

9. Samandari G, Martin SL, Kupper LL, et al. Are Pregnant and Postpartum Women: At Increased Risk for Violent Death? Suicide and Homicide Findings From North Carolina. Matern Child Health J. 2011; 15: 660-669. doi: 10.1007/s10995-010-0623-6.

10. Metz TD, Rovner P, Hoffman MC, et al. Maternal Deaths From Suicide and Overdose in Colorado, 2004-2012. Obstet Gynecol. 2016; 128: 1233.

11. Turner LA, Krame MS, Li S. Cause specific mortality during and after pregnancy and the definition of maternal death. Chronic Disease Canada 2002; 23’31-36.
12. Grigoriadis S, Wilton AS, Kurdyak Paul A, et al. Perinatal suicide in Ontario, Canada: a 15-year population-based study. CMAJ. 2017; 189: E1085-E1092. doi:10.1503/cmaj. 170088

13. Lega I, Maraschini A, D’Aloja P, et al. Maternal suicide in Italy. Arch Womens Ment Health 2019; May 18. Doi:10.1007/s00737019-00997-1.

14. Gissler M, Hemminki E, Lönnqvist J. Suicides after pregnancy in Finland, 1987-94: register linkage study. BMJ. 1996 Dec 7; 313 (7070): 1431-1434.

15. Karalis E, Ulander VM, Tapper AM, Gissler M. Decreasing mortality during pregnancy and for a year after while mortality after termination of pregnancy remains high: a population-based register study of pregnancy-associated deaths in Finland 20012012. BJOG. 2017; 124: 1115-1121. doi:10.1111/1471-0528.14484.

16. Humphrey MD. Maternal mortality trends in Australia. Med J Aust 2016; 205(8)'344-346.

17. Weng S-C, Chang J-C, Yeh M-K, et al. Factors influencing attempted and completed suicide in postnatal women: A populationbased study in Taiwan. Sci Rep. 2016; 6: 25770. doi: 10.1038/srep 25770 .

18. Usuda K, Nishi D, Makino M, et al. Prevalence and related factors of common mental disorders during pregnancy in Japan: a crosssectional study. BioPsychoSocial Medicine. 2016; 10: 17.

19. Suzuki S, Takeda S, Okano T, Kinoshita K. Recent strategies in perinatal mental health care in Japan Hypertension Research in Pregnancy 2018; 6: 11-14. DOI https://doi.org/10.14390/jsshp. HRP2018-006

20. Barker DJ, Osmond C. Infant mortality, childhood nutrition, and ischaemic heart disease in England and Wales. Lancet. 1986; 1: 1077-1081.

21. Barker DJ, Osmond C. Diet and coronary heart disease in England and Wales during and after the Second World War. J Epidemiol Community Health. 1986; 40: 37-44.

22. Barker DJ, Winter PD, Osmond C, Margetts B, Simmonds SJ. Weight in infancy and death from ischaemic heart disease. Lancet. 1989; 2: 577-580.

23. Berry RJ, Li Z, Erickson JD, et al. Prevention of Neural-Tube Defects With Folic Acid in China. China-U.S. Collaborative Project for Neural Tube Defect Prevention. N Engl J Med. 1999; 341: $1485-1490$.

24. Surén $\mathrm{P}$, Roth $\mathrm{C}$, Bresnahan $\mathrm{M}$, et al. Association Between Maternal Use of Folic Acid Supplements and Risk of Autism Spectrum Disorders in Children. JAMA. 2013; 309: 570-577.

25. Bi WG, Nuyt AM, Weiler H, et al. Association Between Vitamin D Supplementation During Pregnancy and Offspring Growth, Morbidity, and Mortality: A Systematic Review and Meta-analysis. JAMA Pediatr. 2018; 172: 635-645.

26. Levine SZ, Kodesh A, Viktorin A, et al. Association of Maternal Use of Folic Acid and Multivitamin Supplements in the Periods Before and During Pregnancy With the Risk of Autism Spectrum Disorder in Offspring. JAMA Psychiatry. 2018; 75: 176-184.

27. Wang M, Li K, Zhao D, et al. The Association Between Maternal Use of Folic Acid Supplements During Pregnancy and Risk of Autism Spectrum Disorders in Children: A Meta-Analysis. Mol Autism 2017; 8: 51. doi:10.1186/s13229-017-0170-8. eCollection 2017. 